\title{
La codification du vote en Suisse (1848-1918), fédéralisme et
} construction du citoyen

In: Genèses, 23, 1996. pp. 76-99.

\section{Résumé}

- Bernard Voutat : La codification du vote en Suisse (1848-1918), fédéralisme et construction du citoyen. Si l'acte de vote est aujourd'hui largement routinisé et naturalisé, l'examen des conflits liés à l'élaboration, au XIXe siècle, d'une législation fédérale sur les droits politiques montre que ce travail de codification juridique a pour enjeu, d'une part, la construction du national où s'opposent, sur le terrain du fédéralisme, partisans et adversaires des particularismes locaux, et, d'autre part, la définition du citoyen marquée par un antagonisme portant sur la maîtrise pratique du processus électoral. Le droit sanctionne ainsi un processus complexe de construction d'un univers politique, où se manifeste une tension permanente entre la territorialisation de l'expression électorale et l'individualisation de l'acte de vote.

\section{Abstract}

Codifying the vote in Switzerland (1818-1918): Federalism and the building of the citizen. Today voting is generally a routine, natural act. However, an examination of the conflicts linked to 19th century development of federal legislation on political rights shows that the task of legal codification involved two challenges : first, the challenge of building the "national" viewpoint in which partisans and adversaries of specific regional identities opposed each other on the issue of federalism, and, secondly, a definition of the citizen marked by antagonism concerning practical control of the electoral process. In this way, the law sanctioned a complex process of building a political world in which constant tension was manifest between the territorializa- tion of electoral expression and the individualization of the act of voting.

Citer ce document / Cite this document :

Voutat Bernard. La codification du vote en Suisse (1848-1918), fédéralisme et construction du citoyen. In: Genèses, $23,1996$. pp. 76-99.

doi : 10.3406/genes.1996.1387

http://www.persee.fr/web/revues/home/prescript/article/genes_1155-3219_1996_num_23_1_1387

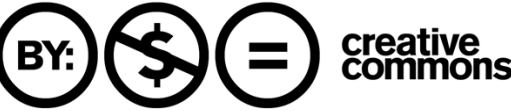


D O S S I E R

Genèses 23, juin 1996, pp. 76-99

\section{LA CODIFICATION}

\section{DU VOTE EN SUISSE}

(1848-1918)

FÉDÉRALISME ET

CONSTRUCTION DU CITOYEN

\section{Bernard Voutat}

1. L'ouvrage d'Erich Gruner constitue toutefois une exception notoire.

Cf. Die Wahlen in den Schweizerischen Nationalrat (1848-1919), Berne.

Francke Verlag, 4 vol., 1978.

Cette étude combine

des questionnements propres

à la science politique et des approches qui relèvent plutôt de l'histoire sociale. Elle s'inscrit donc dans un projet d'histoire sociale du politique et prolonge en cela d'autres travaux réalisés par l'auteur sur le parlement, la formation des partis et des groupes d'intérêts au XIX ${ }^{\circ}$ siècle.

Cf. Die Parteien in der Schweiz.

Berne, Franke, 1969 : Die schweizerische Bundesversammlung, Berne,

Francke, 1966-1970 (5 vol.).

\section{Cette question est largement} développée par Bernard Lacroix. «Ordre politique et ordre social». in Madeleine Grawitz et Jean Leca. Traité de science politique, Paris, PUF, 1985, vol. 1, pp. 469-565.

\footnotetext{
3. Alain Garrigou, Le Vote et la Vertu, Paris, Presses de la Fondation nationale des sciences politiques, 1992, p. 16.
}

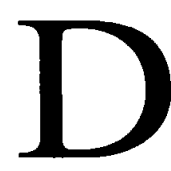
ans l'ensemble, les travaux réalisés en Suisse sur les élections et les votations passent largement sous silence l'acte de vote en lui-même ${ }^{1}$, dimension pourtant capitale, puisqu'elle renvoie à l'identification des ressorts propres à la politisation, et donc aussi à l'invention d'un «ordre politique» qui se construit à travers les usages du vote ${ }^{2}$. Comme le dit fort bien Alain Garrigou, «attentifs à la manière dont les électeurs "font" l'élection, on oublie que l'élection a préalablement "fait" l'électeur» ${ }^{3}$. Nous nous limiterons ici à examiner cette problématique à partir de la législation sur les droits politiques au plan fédéral, qui, sans être un facteur décisif en la matière, sanctionne des usages et représentations antagonistes du vote. Nous pouvons ainsi considérer ce travail de mise en forme juridique, et surtout les conflits qu'il a provoqués, comme révélateurs des enjeux liés au processus de politisation, que nous envisageons ici sous deux aspects spécifiques, associés l'un à l'autre, celui de la (non)nationalisation du vote et celui de l'individualisation du citoyen.

\section{A propos des sources}

Cette étude se fonde sur un dépouillement systématique de la Feuille fédérale entre 1848 et 1918. Il s'agit d'une source imprimée qui réunit les projets de lois soumis aux Chambres fédérales. les messages explicatifs du Conseil fédéral et divers rapports de commissions parlementaires. Nous y avons également trouvé des procès-verbaux de commissions d'enquête à propos de quelques cas importants de fraudes électorales survenus dans les cantons du Tessin et du Valais. Ces documents permettent de dresser un premier aperçu du travail législatif sur les droits politiques réalisé 
au niveau fédéral durant la période considérée. Une étude plus complète devrait toutefois s'élargir à d'autres sources (débats parlementaires, presse, jurisprudence) et, surtout, s'étendre à l'intense travail de codification juridique qui s'effectue dans les vingt-deux cantons de la Confédération, où sont par ailleurs dispersées d'autres sources (procès verbaux d'assemblées électorales, rapports concernant des irrégularités dans le déroulement des scrutins, etc.) autorisant une analyse plus fine des pratiques électorales effectives, sources qui n'ont pour l'heure que peu retenu l'attention des politistes et historiens.

\section{Naturalisation et routinisation de l'acte de vote}

Dans son Message du 9 avril 1975 à l'Assemblée fédérale, le Conseil fédéral porte un jugement sévère concernant l'état de la législation sur les droits politiques : «Les dispositions qui régissent actuellement les droits politiques du citoyen suisse - il s'agit principalement de six lois concernant l'exercice du droit de vote ainsi que du droit d'initiative et de référendum - se trouvent disséminées dans de nombreux actes législatifs dont certains datent des années septante du siècle dernier. Ce partage entre plusieurs textes spéciaux ainsi que de multiples renvois font qu'il est difficile d'avoir une vue d'ensemble sur cette matière législative ; comportant des lacunes, celle-ci est en outre surannée» ${ }^{4}$. Ce constat général amène alors le Conseil fédéral à préconiser une révision d'ensemble, afin «de regrouper dans une seule loi toutes les dispositions touchant les droits politiques», et ceci en vue «d'unifier dans toute la mesure du possible, sur le plan fédéral, les prescriptions relatives aux droits politiques». Certes, reconnaissant que l'organisation des votations et des élections incombe aux cantons, le Message ajoute qu'il est nécessaire de «tenir compte de certaines particularités cantonales» pour que le projet de loi soit «acceptable dans l'ensemble du pays»'.

D’une manière générale, la procédure de consultation fait ressortir un large accord concernant la nécessité d'une révision législative destinée à harmoniser le droit en la matière au niveau fédéral. Outre la complexité de la situation résultant de la dualité d'un droit fédéral réduit à quelques principes généraux et de législations cantonales très diversifiées, il est invoqué le fait que l'organisation «technique» des élections et des votations fédérales doit également faire l'objet de dispositions homogènes au plan national. En fait, cette révision législative fut assez
4. Il s’agit des textes législatifs suivants : loi fédérale du 19 juillet 1872 sur les élections et votations fédérales (qui remplace la première loi fédérale du 21 décembre 1850) : loi fédérale du 17 juin 1874 concernant les votations populaires sur les lois et arrêtés fédéraux : loi fédérale du 14 février 1919 concernant l'élection du Conseil national : loi fédérale du 23 mars 1962 concernant le mode de procéder pour les initiatives populaires relatives à la révision de la constitution (qui remplace la loi fédérale du 27 janvier 1892) ; loi fédérale du 8 mars 1863 répartissant entre les cantons les députés au Conseil national : loi fédérale du 25 juin 1965 instituant des facilités en matière de votations et d'élections fédérales.

5. Message du Conseil fédéral à l'Assemblée fédérale du 9 avril 1975 concernant le projet d une loi fédérale sur les droits politiques. Féuille fédérale. 1975. I. pp. 1337 sq. 


\section{O S S I E R}

consensuelle. Les articles consacrés à l'octroi du droit de vote, à ses conditions d'exercice ou encore aux modalités pratiques de son organisation n'ont guère suscité de remous au Parlement fédéral. Seules les formations politiques de gauche ont contesté les restrictions apportées à la démocratie directe (élévation du nombre de signatures et du temps requis pour leur récolte), mais elles subirent un échec à l'occasion de la votation populaire.

Cette loi de 1976, outre qu'elle réalise un accord sur la question autrefois sensible de la capacité électorale, consacre dans l'ordre juridique une distinction désormais bien établie entre deux types de dispositions en matière d'exercice des droits politiques. Les unes, qui concernent principalement le dispositif concret par lequel le vote est aménagé (lieu du vote, matériel remis au citoyen, aménagement du bureau de vote, etc.), sont désormais largement considérées comme simplement «techniques» ou «formelles», par opposition à d'autres qui, en raison de leurs effets sur la «vie politique», sont directement investies d'une signification politique (modes de scrutin, règles relatives à l'initiative et au référendum). Cette façon de voir le vote, sanctionnée par la loi, est donc révélatrice du fait que l'acte lui-même, dans ce qu'il a de plus pratique, est désormais pourvu d'une très grande évidence, que le déroulement de la procédure électorale a perdu son caractère conflictuel pour acquérir le statut de simple dispositif «formel» et «routinisé» - assurant la neutralité et la sincérité des scrutins, respectant donc la volonté du corps électoral et des différentes «opinions» qui composent ce qu'il est aujourd'hui convenu d'appeler l'«opinion publique». Or, cette évidence perd singulièrement de sa solidité si l'on considère que l'un des principaux enjeux des luttes politiques dans le long processus de codification juridique de l'acte de vote en Suisse résidait précisément dans la question de l'organisation concrète des scrutins, dont l'importance égalait, sinon dépassait d'autres volets comme l'attribution de la capacité électorale ou la détermination du mode de scrutin, au point de constituer un obstacle majeur à l'élaboration d'une législation nationale sur les droits politiques'.

Aujourd'hui, pourtant, les dispositions des différentes législations sur les droits politiques retenant le plus l'attention des historiens sont celles qui concernent les modes de scrutin. Une abondante littérature existe en Suisse sur les débats politiques entourant l'introduction du système pro- 
portionnel et de la démocratie directe (référendum et initiative), en tant qu'ils révèlent des conceptions antagonistes de la représentation et donc aussi de la démocratie comme régime politique. En revanche, peu d'ouvrages sont directement consacrés aux règles applicables au déroulement proprement dit des élections - encore moins, faut-il le préciser, à ce déroulement lui-même, alors que ce problème est au cœur du débat politique durant tout le $\mathrm{XIX}^{\mathrm{e}}$ siècle $^{7}$, et qu'il est, pour cette raison déjà, au moins aussi révélateur que ne le sont les conflits de doctrine entre différentes approches de la représentation populaire ou encore de la capacité électorale.

Cette lacune dans l'historiographie est, à nos yeux, en partie imputable à une projection dans le passé d'une représentation contemporaine largement naturalisée de l'acte de vote, qui repose sur une opposition actuelle entre dispositions «techniques» et «politiques». En réalité, cette distinction s'élabore sur la longue durée, au cours du XIX ${ }^{\mathrm{e}}$ siècle, et ne s'impose que dans le premier quart du $\mathrm{xx}^{\mathrm{e}}$ siècle. Si l'organisation pratique des scrutins est alors une question éminemment conflictuelle, c'est qu'elle affecte le mode même par lequel les entrepreneurs politiques s'engagent dans l'activité de conquête des suffrages, tout en sanctionnant et en objectivant des représentations concurrentielles du citoyen et de son rapport au politique.

L'acte de vote : un objet marginal

Les particularités du système politique helvétique (structure fédéraliste, démocratie représentative associée à des mécanismes de démocratie directe, fréquence très élevée des scrutins, complexité des objets soumis au vote) ont largement contribué au développement de recherches sur les institutions et comportements politiques. Nous disposons ainsi de nombreux travaux relevant soit de l'histoire constitutionnelle, centrée sur les innovations institutionnelles marquant la scansion des régimes politiques ; soit de l'histoire politique, consacrée pour l'essentiel à l'analyse des différents courants, programmes et actions des forces politiques engagées dans le processus de construction de l'État fédéral ; soit enfin de l'histoire des idées, étudiant plus spécifiquement les fondements doctrinaux de la démocratie suisse. Le droit public a produit de nombreux traités sur les systèmes électoraux (fédéraux et cantonaux) et sur les mécanismes de démocratie directe, et ceci dès leur institutionnalisation dans l'ordre juridique au XIX ${ }^{\mathrm{e}}$ siècle. Outre les commentaires consacrés au fonctionnement et aux conditions d'exercice des droits politiques en Suisse, ces études élargissent fréquemment leur propos à une évaluation philosophique et normative de la nature même du régime politique mis
7. Dans un message datant de 1883 , le Conseil fédéral reconnaît d'ailleurs lui-même que «la loi de 1872 sur les élections et votations fédérales a fait peut-être plus qu'aucun autre décret du législateur suisse l'objet, on peut dire dès son origine, de critiques de la part du public et de discussions dans le sein des Conseils de la Confédération». Message du Conseil fédéral à l'Assemblée fédérale du 30 octobre 1883 concernant le projet d'une loi fédérale sur les élections et votations fédérales, Feuille fédérale, 1883, IV, p. 183. 


\section{O S S I E R}

8. Cf. notamment Roland Ruffieux. "Les données de l'histoire constitutionnelle», in Manuel du système politique de la Suisse, Berne. P. Haupt, vol. 1, 1983. pp. 119-209: Aloïs Riklin et Silvano Möckli, «Ideengeschichtlicher Kontext". in ibid. pp. 9-118: Leonard Neidhart, Plebiszit und pluralitäre Demokratie : eine Analyse der Finktion des schweizerischen Gesetzesreferendums, Bern, Francke, 1970 ; Yannis Papadopoulos (éd.), Elites politiques et peuple en Suisse. Analyse des votations fédérales: 1970-1987, Lausanne, Réalités sociales. 1994 ; Jean-Daniel Delley, L'Initiative populaire en Suisse. Mythe et réalité de la démocratie directe, Lausanne, L'Age d'Homme, 1978 ; Roland Ruffieux (éd.), La Démocratie référendaire en Suisse au $X X^{e}$ siècle, Fribourg, Éditions Universitaires, 1972 ; Jean-Bernard Racine, «La Suisse dans son intériorité : une géographie des opinions", in Jean-Bernard Racine et Claude Raffestin (éd.), Nouvelle géographie de la Suisse et des Suisses, Lausanne. Payot, 1990, vol. 2. pp. 543-564; Hanspeter Kriesi, Citoyenneté et démocratie directe. Compélence, participation et décision des citoyens et citoyennes suisses, Zürich. Seismo, 1993. en place en 1848, considéré sous l'angle de ses avantages, de ses inconvénients et de ses fondements en regard d'une théorie de la démocratie et de la souveraineté populaire. A partir d'une typologie formelle des systèmes politiques, les juristes classent alors la Suisse comme une démocratie semi-directe associant, par le biais de l'élection et de la votation, une dimension représentative et plébiscitaire. En science politique, l'attention s'est également portée sur l'élection et la votation, à partir des «fonctions» que remplissent ces institutions et des conséquences que leur usage entraîne au niveau de la répartition du pouvoir, notamment dans le processus de décision. Le vote n'est alors que rarement appréhendé en tant que tel, puisque l'analyse se limite à saisir le rôle des instruments de démocratie directe du point de vue des acteurs (partis, groupes d'intérêts, mouvements sociaux) et de la fonctionnalité du système politique, en regard des contraintes ou opportunités qu'ils permettent et des effets (de légitimation, de blocage ou d'innovation) qu'ils induisent. Enfin, la sociologie des comportements politiques a également donné lieu à de nombreux travaux, notamment sur les niveaux de participation, l'abstentionnisme (jugé généralement très important en Suisse), les «motivations» du citoyen, les déterminants sociaux des attitudes en matière électorale et à l'occasion des votations. Compte tenu de la diversité culturelle qui caractérise la Suisse, une attention particulière est accordée à la territorialité des opinions, soit à travers des analyses écologiques du vote, soit plus généralement dans les commentaires politiques. Une hésitation centrale se manifeste toutefois dans ces écrits, qui tantôt adoptent une perspective plutôt descriptive (et simultanément normative) mettant l'accent sur les dangers que les clivages (linguistiques, confessionnels, cantonaux, etc.) issus de certains scrutins font courir à la cohésion du pays, tantôt érigent ces clivages en facteurs explicatifs des comportements politiques, mais de façon peu ou pas problématisée. En fait, bien que les spécificités du système politique suisse soient souvent invoquées pour le considérer comme un «cas à part», les analyses, dans leur contenu comme dans leurs orientations générales, ne sont pas très éloignées de la production scientifique élaborée ailleurs sur le vote. Finalement, l'acte en lui-même et les usages dont le vote est l'objet sont assez largement marginalisés dans les travaux réalisés par les sciences sociales en Suisse. ${ }^{8}$

\section{La codification du vote et ses enjeux}

Inauguré en 1798, restreint en 1803 , puis aboli ou fortement limité en 1815, le suffrage universel (masculin) réapparaît dans les cantons suisses en 1830 , moyennant parfois plusieurs restrictions (cens, élection à deux degrés, cooptation, etc.), et tout ceci dans des systèmes électoraux d'une complexité que notre accoutumance au vote rend aujourd'hui étonnante. Mais c'est plutôt la création de l'État fédéral, à la faveur des révolutions de 1848 en Europe, qui retient l'attention des historiens du vote en 
Suisse ${ }^{9}$. Ceux-ci soulignent alors l'extension de la démocratie («testée» dans certains cantons), sous la poussée des «démocrates progressistes» d'abord, puis des socialistes, à travers le droit de référendum (1874), d'initiative (1891) et l'introduction du scrutin proportionnel au niveau fédéral pour l'élection du Conseil national (1919). Cette périodisation classique, bien que commode, «privilégie les coupures institutionnelles, juridiques et formelles (...) ; elle tend à effacer la continuité du pouvoir d'un régime à l'autre et néglige les mécanismes de transmission de l'autorité» ${ }^{10}$. Comme le souligne Gérald Arlettaz à propos de la première moitié du XIX ${ }^{e}$ siècle (mais le jugement pourrait être étendu à tout le siècle), «les personnalités politiques, qui passent aisément d'un régime politique à l'autre, sont le témoignage de structures oligarchiques et restent très attachées à leurs privilèges de caste» ${ }^{11}$. En outre, en oscillant entre une perception de la construction démocratique «par en bas», en terme de conquête "populaire», ou "par en haut», c'est-à-dire comme résultat de l'activité étatique, cette conception de l'«essor démocratique» écarte de l'analyse les conditions, les logiques et les médiations (pratiques et symboliques) intervenant dans le processus complexe de la politisation.

Or, l'un des aspects singuliers de la législation en Suisse concernant les droits politiques réside dans la très large autonomie laissée aux cantons, de sorte qu'à un droit fédéral résiduel (qui plus est, applicable aux seuls scrutins fédéraux) s'ajoutent vingt-deux législations cantonales, fort différentes les unes par rapport aux autres et qui, dans la deuxième moitié du XIX ${ }^{\mathrm{e}}$ siècle, connaissent des transformations importantes ${ }^{12}$. C'est dire la difficulté de retracer exhaustivement l'histoire de l'ensemble de cette période sous son aspect juridique, même si, par-delà les spécificités cantonales, on est amené à identifier les logiques fondamentales qui nous intéressent ici.

Les premières élections au Conseil national de 1848 se sont déroulées selon les modalités et principes appliqués précédemment dans les cantons. Deux ans plus tard, en prévision du scrutin fédéral de 1851 , une loi est votée ${ }^{13}$, qui se borne à fixer le mode de scrutin et à régler la question alors sensible du découpage des circonscriptions électorales. Pour le reste, le texte reste laconique, voire flou, et même contradictoire. Posant le principe général du suffrage universel masculin dès l'âge de vingt ans, cette loi le vide d'emblée d'une partie de
9. Cet événement marque l'avènement de la «Suisse moderne» en ce qu'il instaure au plan national lc suffrage «universel» pour l'élection à la Chambre basse (le Conseil national).

10. Georges Andrey,

«La quête d'un État national». in Nouvelle histoire de la Suisse et des Suisses, Lausanne, Payot.

1986 (2c ćd.). p. 574.

11. Gérald Arlettaz. «La démocratie au temps des Républiques. Idéologies et mentalités politiques dans la société suisse (1815-1848)». Etudes et sources, Berne, Archives fédérales suisses, n9. 1983. p. 10.

12. Cette diversité se mesure également à la varićté des régimes politiques en vigueur dans les cantons suisses. Selon une typologie classique. on distingue les régimes de «démocratie pure», où les élections et votations se déroulent dans des Landsgemeinde regroupant en un même licu l'ensemble des citoyens jouissant des droits politiques, les régimes de «démocratie représentative» et les régimes de «démocratie semi-directe», combinant une dimension représentative et des institutions comme l'initiative. le référendum ou le veto.

13. Loi fédérale du 21 décembre 1850 concernant les élections des membres du Conseil national, Recueil officiel des lois fédérales $(R O)$, III. 1850. pp. 205 sq.. qui introduit le scrutin majoritaire, pluri-nominal à trois tours en vigueur jusqu'en 1919. moment de l'introduction du système proportionnel. 


\section{O S S I E R}

Histoire politique, histoire du politique II

Bernard Voutat

La codification du vote en Suisse $(1848-1918)$ fédéralisme

et construction du citoyen son contenu en subordonnant l'accès aux urnes au respect de la législation du canton dans lequel le citoyen a élu son «domicile». Le droit de vote est ainsi accordé pour les élections fédérales aux mêmes conditions que pour les élections cantonales. De même, les cantons demeurent pleinement souverains pour décider de la procédure électorale proprement dite. Il leur incombe notamment, selon l'article 12 de la loi, de décider «si les élections pour le Conseil national ont lieu par commune ou au chef-lieu des cercles électoraux établis pour les élections des autorités cantonales, ou dans d'autres assemblées, et si ces élections se font au scrutin secret, ou publiquement, soit à main levée».

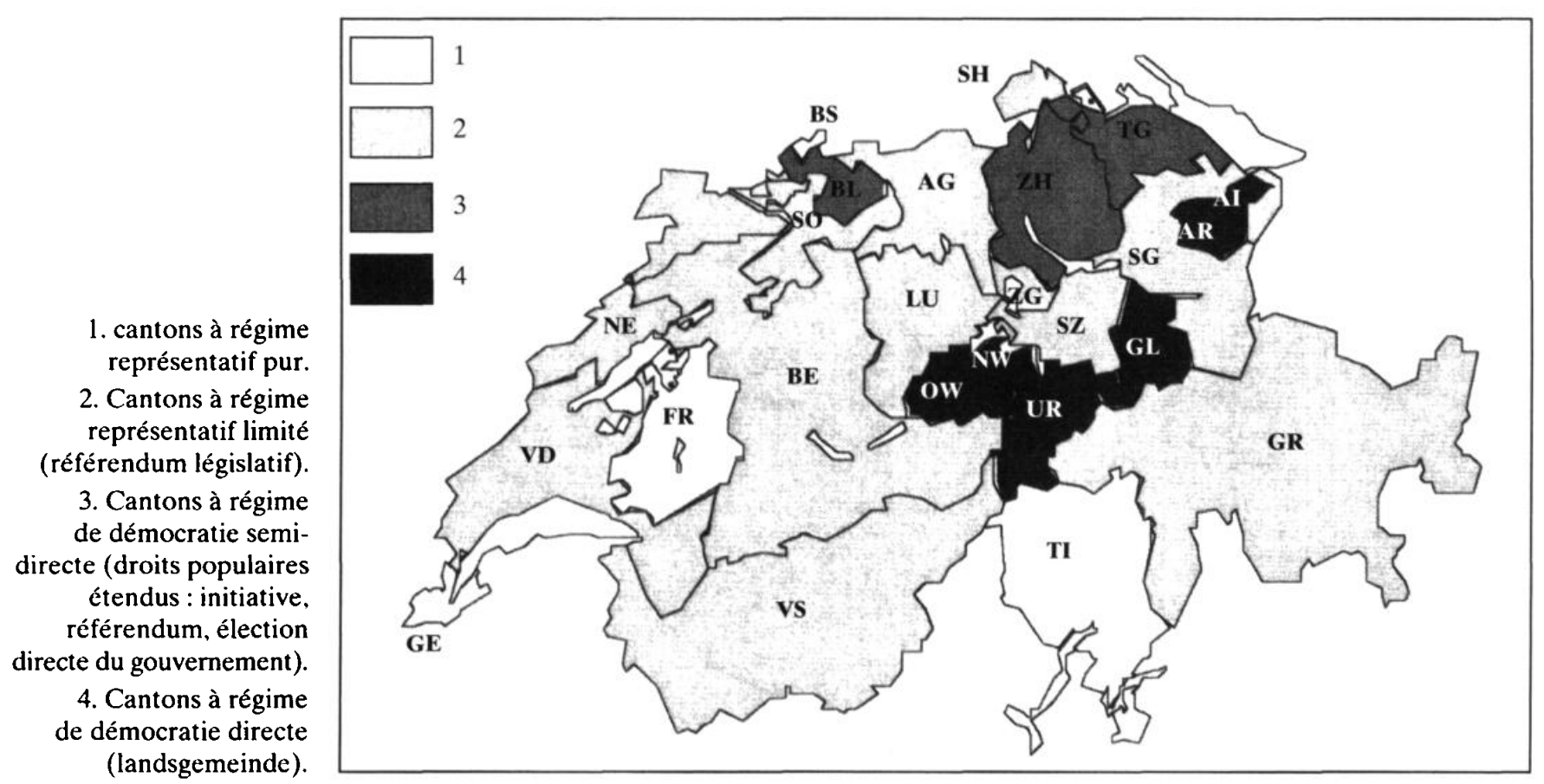

14. Par exemple, cinq cantons accordent le droit de vote à un âge inférieur à 20 ans jusqu'au début des années 1870 (Grisons 17 ans ; Appenzell, Glaris et Schwyz 18 ans; Zoug 19 ans).

15. La notion de «domicile politique» n’est précisée au plan fédéral que dans une loi spéciale adoptée en 1951 (cf. $R O, 1952$, pp. 69 sq.).
De fait, les pratiques se révèlent très hétérogènes ${ }^{14}$. En particulier, la clause de domicile est inégalement interprétée dans les cantons ${ }^{15}$. Certains n'accordent les droits politiques qu'aux citoyens «originaires» d'une commune du canton de domicile, à l'exclusion des «confédérés» (c'est-à-dire des Suisses originaires d'un autre canton, mais «résidant» dans le canton de domicile). D'autres exigent une clause de réciprocité pour octroyer le droit de vote aux confédérés originaires d'un autre canton. D'autres, enfin, excluent les confédérés du droit de vote, mais l'accordent à leurs ressortissants domiciliés à l'«étranger», c'est-à-dire aussi en Suisse. Cet aspect des législations électorales cantonales concernant 
l'identification des citoyens montre que le vote est relié étroitement à une logique communautaire, qui sanctionne une distinction entre le domicile, assimilé à une simple résidence, et l'origine, marquant l'appartenance à une commune et au canton dont elle dépend ${ }^{16}$. Mais il révèle également une logique plus directement politique, qui renvoie, d'une part, à la question de l'appartenance nationale d'une population instable de confédérés - tantôt exclue du corps des citoyens, tantôt incluse - mais qui tient aussi, d'autre part, au contrôle des registres électoraux, voire, plus prosaïquement, à leur manipulation par déplacement d'une partie de l'électorat flottant d'un lieu à un autre ${ }^{17}$.

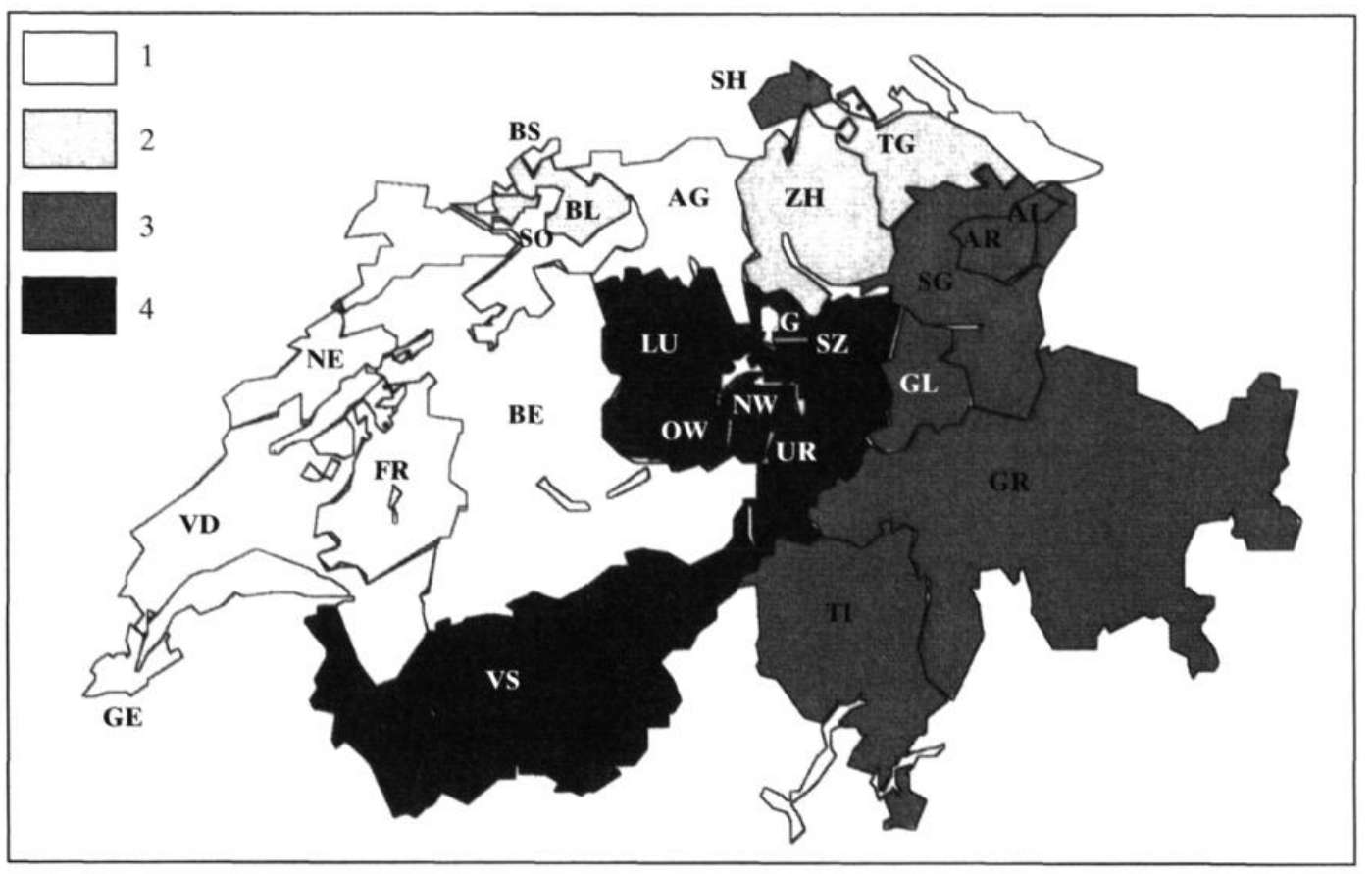

1. Cantons à dominante radicale.

2. Cantons à dominante radicale-démocrate. 3. Cantons où existe un équilibre entre partis. 4. Cantons à dominante conservatrice catholique.

La notion de «bourgeoisie» et la question du domicile

Les interprétations divergentes de la notion de «domicile» sont liées, en Suisse, au fait que celle-ci renvoie tantôt à la commune où le citoyen, au bénéfice d'un permis de séjour ou d'établissement, réside effectivement, tantôt à la commune dont il est originaire, qui est dite commune «bourgeoise». Celle-ci tire ses racines de l'Ancien Régime, où les agglomérations, jouissant d'une certaine autonomie, étaient administrées par les «bourgeois». L'origine se transmet de père en fils (les femmes adoptent à leur mariage celle de leur mari) et doit être distinguée du lieu de naissance. Elle confère à l'individu un «droit de bourgeoisie», notamment celui de participer à la gestion des affaires communales, de bénéficier des biens «bourgeois» (bois et pâturages), ou encore de solliciter l'assistance. Le lieu d'origine constitue donc, dans les recensements

16. Sur l'identification, cf. Michel Offerlé, «L'électeur et ses papiers», Genèses, no 13, 1993, pp. 29-53.

En outre, la logique communautaire que nous évoquons s'apparente ici à ce que Max Weber appelle "communalisation», soit «une relation sociale lorsque, et tant que. la disposition de l'activité sociale se fonde [...] sur le sentiment subjectif des participants d'appartenir à une même communauté». Cf. Max Weber, Économie et société, Vol. 2, Paris, Plon, 1995, p. 78. 


\section{O S S I E R}

17. Sur cette question,

cf. Michel Offerlé, «La nationalisation de la citoyenneté civique en France", papier présenté au colloque de l'Institut Universitaire Européen de Florence (20-22 avril 1995) sur le thème «How did they become voters. The history of franchise in modern curopean representational systems», $16 \mathrm{p}$. Pour la Suisse, cf. Muriel Surdez, «Quand les frontières se font statistiques. La question de la constitution d'un espace national considérée à travers le prisme des recensements", Revue suisse dhistoire, vol. $45, \mathrm{n}^{\circ} 1$, 1995, pp. 63-79.

18. Erich Gruner fournit une statistique détaillée pour toute la période, tout en indiquant que les chiffres ne sont véritablement fiables qu'à partir du premier recensement fédéral de 1881. Cf. Die Wahlen in den Schweizerischen Nationalrat (1848-1919), vol. 3, op. cit., pp. 345 sq.

19. Cf. pour le détail,

Fenille fédérale, 1885, II, pp. 4 sq. ou dans certaines pratiques administratives, une catégorie statistique distincte du lieu de résidence ou de naissance. Il institue au plan symbolique un principe d'appartenance territoriale, qui est sanctionné par l'État et son administration. Alors qu'au début du $\mathrm{XIX}^{\mathfrak{c}}$ siècle résidence et origine se confondaient fréquemment, le brassage des populations consécutif à la révolution industrielle a eu comme conséquence une distorsion toujours plus discriminante entre «bourgeois» et "confédérés», c'est-à-dire citoyens suisses résidant ailleurs que dans leur lieu d'origine. La «bourgeoisie», comme institution, enferme une charge symbolique importante, dans la mesure où elle fixe un principe d'identification des personnes dont dépend parfois la capacité électorale. Celle-ci reste donc étroitement associée à une logique communautaire, définie par l'appartenance à la commune «bourgeoise» qui est progressivement supplantée par la commune politique ou «municipalité», cette dernière réalisant l'égalité entre tous les citoyens.

Outre les critères d'âge, de droit de cité et de domicile, qui constituent, selon un terminologie propre au droit public, les conditions objectives de la capacité électorale, les cantons appliquent de façon diversifiée d'autres causes d'exclusion des droits politiques, relevant cette fois-ci des conditions subjectives, qui font dépendre l'exercice et la jouissance de ces droits de certaines circonstances liées à la position sociale de l'individu (faillite, assistance, statut dépendant) ou à son comportement (interdiction civile, condamnation pénale, interdiction d'auberge). Les autorités locales disposent ainsi d'une marge de manœuvre supplémentaire dans le contrôle de l'électorat.

De fait, le rapport entre l'électorat potentiel, composé des citoyens suisses, résidant dans un canton, âgés de 20 ans, et l'électorat réel, regroupant les citoyens effectivement inscrits dans les registres électoraux, est fortement tributaire des pratiques qui se mettent en place au niveau cantonal concernant les inscriptions dans les registres électoraux, et ceci nonobstant le principe de l'inscription d'office au «domicile» introduit dans la loi fédérale de 1872 sur les élections au Conseil national. Ainsi, dans certains endroits comme au Tessin ou, dans une moindre mesure, à Genève, ce rapport atteint des proportions très supérieures à $100 \%$ jusqu'en 1918, dans la mesure où sont également inscrits dans les registres électoraux les citoyens qui ne résident pas dans le canton, mais qui en sont «originaires». Par contraste, il n'est pas rare que certains cantons écartent des urnes plus du quart des électeurs durant la même période, soit en n'accordant le droit de vote qu'aux citoyens «bourgeois» résidant dans le canton, soit par cumul des diverses restrictions apportées à la capacité électorale sous l'angle de ses conditions subjectives ${ }^{18}$. En 1885 , le Conseil fédéral estime à 100000 le nombre de citoyens exclus du droit de vote, sur un électorat potentiel de 730000 citoyens suisses, de vingt ans, domiciliés dans le pays ${ }^{19}$. 
En définissant les qualités (notamment l'appartenance à une collectivité) ou a contrario les vertus (indépendance, intégrité, moralité) requises pour accéder au statut de citoyen, les dispositions régissant l'octroi ou le retrait des droits politiques fournissent du même coup un éclairage sur les représentations dont ils sont investis $^{20}$. Celles-ci ne sont cependant pas indépendantes des pratiques, donc des conditions concrètes dans lesquelles ces droits sont effectivement exercés.

S'agissant du déroulement proprement dit des scrutins, les usages du vote, qui résultent d'une tradition «démocratique» plus ou moins établie selon les endroits, s'avèrent là également très hétérogènes. Au milieu du $\mathrm{XIX}^{\mathrm{e}}$ siècle, au moment même de l'avènement de l'État fédéral et dans les années qui suivent, les élections demeurent toutefois fortement marquées par une logique communautaire. Elles se déroulent la plupart du temps au sein d'assemblées publiques encadrées par des notables qui contrôlent dans une large mesure la succession des opérations ${ }^{21}$. On comprend ainsi que des questions aujourd'hui secondaires, comme la fixation de la date et du lieu de réunion, sa durée, la tenue des registres électoraux ou encore les modalités concrètes d'expression du vote revêtent alors une importance toute particulière. Comme le résume bien Gruner, «les procédés les plus courants utilisés pour étouffer une possible ou réelle majorité de la part de l'opposition, ont consisté à organiser, en des endroits reculés et au vote à main levée, des assemblées électorales aussi vastes que possible et à en assurer le résultat par le choix approprié d'un bureau à couleur politique déterminée» 22 .

Certes, cette appréciation générale masque les diversités cantonales. Certains cantons connaissent déjà le système de l'urne et entourent l'acte de vote d'une garantie, au moins formelle, de secret. Ainsi, par exemple, dans le canton de Vaud, l'électeur remplit personnellement son bulletin dans un lieu désigné à cet effet et séparé, mais à l'intérieur de la salle, puis le remet plié au Président du bureau, qui le glisse dans l'urne ${ }^{23}$. Des procédures analogues existent à Neuchâtel, Bâle et Schaffhouse. Dans les autres cantons, le vote est «apparemment secret» 24 , mais en réalité souvent ouvert. Entendons par là que le scrutin, réputé secret, s'effectue en assemblée, que des pressions peuvent donc s'exercer sur l'électeur et que toute une série de procédés sont mis en œuvre pour
20. Cf. un développement sur ce problème dans le Message du Conscil fédéral du 2 octobre 1874 à la haute Assemblée fédérale concernant la loi sur le droit de vote des citoyens suisses, Feuille Fédérale. III. 1874. pp. 40 sq.

21. Avec Max Weber, nous définissons les notables comme des personnes qui, «par leur situation économique. sont en mesure, à titre de profession secondaire, de diriger et d'administrer effectivement de façon continue un groupement quelconque, sans salaire ou contre un salaire honorifique. [et qui] qui jouissent d'une estime sociale [...] de sorte qu'elles ont la chance d'occuper des fonctions dans une démocratie directe formelle. en vertu de la confiance de ses membres, d'abord par acte volontaire, puis à la longue par tradition. Les notables peuvent vivre pour la politique sans devoir vivre d'elle". Cf. Max Weber, Économie et société, op. cil.. Vol. 1, pp. 378-379.

22. Erich Gruner, Die Wahlen in den Schweizerischen Nationalrat (1848-1919), op. cit., Vol. 2, p. 1085.

23. Cette procédure, définie dans une loi de 1832 , sera complétée en 1881 par l'introduction de l'enveloppe, afin de renforcer le secret du vote.

24. «Scheinbar geheim» selon l'expression de Gruner, Die Wahlen in den Schweizerischen Nationalrat (1848-1919), vol. 1, op. cit., p. 112. 


\section{O S S I E R}

\author{
Histoire politique, \\ histoire du politique II \\ Bernard Voutat \\ La codification du vote en Suisse \\ (I848-19I8) \\ fédéralisme \\ et construction du citoyen
}

25. Cette institution fonctionne du reste encore à l'heure actuelle dans quelques cantons de la Suisse centrale, mais son autorité est limitée aux affaires qui relèvent de leur compétence.

26. Ces catégories politiques sont loin de désigner des réalités homogènes et unifiées. Disons, pour simplifier, que les «radicaux», porteurs d'un projet politique national et présents sur l'ensemble du territoire helvétique, sont favorables à une centralisation législative au niveau fédéral, faisant écho à la fois aux nécessités économiques invoquées par la bourgeoisie industrielle et financière, et aux exigences démocratiques soutenues par un courant socialisant issu d'une classe ouvrière en voie de formation. Les socialistes apparaissent plus tardivement. Favorables eux aussi à l'élargissement des droits politiques, ils combattent dès les années 1880 le scrutin majoritaire, qui les tient pratiquement à l'écart du Parlement fédéral jusqu'à l'introduction du système proportionnel, suite à une initiative qu'ils ont largement soutenue. Les «libéraux», héritiers des révolutions de 1830 , s'opposent aux précédents sur la question de la préservation des particularismes locaux et s'efforcent dès lors de limiter l'extension des droits démocratiques, fidèles en cela à la pensée libérale que développent certaines notabilités locales (avocats, juges, propriétaires fonciers). Le courant "conservateur», étroitement associé à l'Église catholique dans les petits cantons ruraux de la Suisse centrale, s'accommode dans ses fiefs du système démocratique, qu'il parvient à maîtriser

par un encadrement massif de l'électorat et par une lutte constante visant, au nom de la tradition, à éviter toute centralisation législative au niveau fédéral.

27. Message du Conseil fédéral du 24 juin 1872 touchant un projet de loi sur les élections et les votations fédérales, Feuille Fédérale, I, 1872, p. 826 . déchiffrer les votes (bulletin remis ouvert au Président du bureau, couleur ou texture du papier reconnaissables, manipulation des urnes, formes d'écritures, etc.). Plusieurs cantons, la plupart conservateurs, maintiennent le système du vote à main levée dans des Landsgemeinde ${ }^{25}$ ou des assemblées analogues. Bref, les vingt premières années de la Confédération sont marquées par une procédure électorale où le vote, vu sa publicité, s'exprime sous un «contrôle notabiliaire». Dans les différents cantons, cette période est particulièrement prolixe en lois électorales et la question du secret, à côté de celle des conditions d'octroi des droits politiques, constituent les enjeux principaux qui opposent conservateurs, libéraux et radicaux selon des modalités changeantes, étroitement liées aux rapports de force politiques qui se nouent au niveau local ${ }^{26}$. Cet antagonisme, qui se manifeste alors sur le plan de la codification de l'acte de vote, porte sur la maîtrise d'une pratique qui tend à se généraliser, mais qui est inséparable du sens attaché à la notion même de citoyen/électeur, en tant que membre d'une collectivité et dépositaire d'une voix.

\section{Transformations sociales et non-nationalisation du vote en Suisse}

Durant toute la deuxième moitié du XIX ${ }^{\mathrm{e}}$ siècle, la codification juridique de l'acte de vote s'est poursuivie à un rythme soutenu, mais principalement dans les parlements des différents cantons où ce problème est fréquemment mis à l'ordre du jour. En 1872, toutefois, le Conseil fédéral préconise l'amorce d'une unification de la législation sur les votations et les élections, tout en restant fidèle au principe général de l'autonomie cantonale. A ses yeux, «il est évident que l'élaboration d'une loi électorale fédérale rencontrerait bien des difficultés. Dans la plupart des cantons et dans les plus grands, l'organisation des élections et des votations est tellement compliquée qu'il ne paraît pas convenable d'y ajouter encore, par voie de législation fédérale, de nouveaux rouages qui nécessairement devraient différer beaucoup de ceux du plus grand nombre de cantons» ${ }^{27}$. Sont notamment touchées les dispositions concernant la majorité civique, l'exclusion du droit de vote, en particulier la clause de domicile, ainsi que la question de l'organisation concrète des élections et des votations. 
La loi fédérale, qui est adoptée par les chambres le 19 juillet 1872 , s'en tient donc à quelques principes généraux $^{28}$. Elle introduit désormais l'obligation pour les cantons de fixer la majorité civique à vingt ans. En revanche, elle leur laisse la faculté de déterminer les conditions subjectives attachées à l'exercice et à la jouissance des droits politiques. Cette loi, en outre, accorde le droit de vote en matière fédérale aux confédérés «domiciliés» dans un autre canton que le leur et pose le principe de l'inscription d'office sur les registres électoraux. Enfin, elle adopte le principe du vote secret (applicable aux seuls scrutins fédéraux) en lieu et place du vote à main levée en vigueur dans les quelques cantons où subsiste la Landsgemeinde.

En fait, ces quelques dispositions sont loin de conduire à la réduction des disparités cantonales. Les discriminations envers l'électorat flottant des confédérés, souvent privés des droits politiques au niveau cantonal ou communal, de même qu'envers les faillis et personnes bénéficiant de l'assistance publique, se maintiennent là où, du reste, elles existaient déjà - soit principalement dans les cantons tenus par une majorité conservatrice. Quant au secret du vote, son effectivité demeure précaire, notamment lors d'élections très disputées où les fraudes et irrégularités sont monnaie courante, mais plus généralement en fonction d'une structure sociale qui demeure largement communautaire, donc dominée par des notables locaux assurant régulièrement un contrôle sévère de la procédure électorale. Le Parlement fédéral (sous hégémonie radicale durant toute la période considérée) s'efforce alors à plusieurs reprises d'unifier les droits politiques au niveau national. Il se heurte toutefois à une coalition hétérogène qui parvient, en invoquant l'autonomie des cantons en la matière et le respect de la structure fédéraliste du pays, à faire échouer les projets de loi proposés par les autorités fédérales ${ }^{29}$.

Le débat à propos de la centralisation, au niveau fédéral, des règles applicables à l'occasion des scrutins se joue en partie sur le problème de l'appartenance (nationale, cantonale et communale) des citoyens appelés à voter. C'est ainsi, par exemple, que Pidou, député libéral à l'Assemblée constituante du canton de Vaud, peut être amené à considérer que «mettre les Vaudois et les confédérés sur le même pied ne signifierait rien moins que la transformation des cantons en de simples divisions terri-
28. Recueil officiel des lois fédérales, $R O, 1872$, pp. 770 sq.

29. En 1875 et 1877, deux projets de loi destinés principalement à harmoniser la législation en matière d'exclusion des droits politiques frappant les confédérés échouent en votation populaire suite au dépôt d'un référendum. Cf. le Message du Conseil fédéral du 2 octobre 1874 à la haute Assemblée fédérale concernant la loi sur le droit de vote des citoyens suisses, Feuille Fédérale, III, 1874, pp. 34 sq., ainsi que le Message du Conseil fédéral du 25 octobre 1876 concernant le projet de loi sur les droits politiques des Suisses établis et en séjour et la perte des droits politiques des citoyens suisses, Feuille Fédérale, III, 1876, pp. 75 sq. En 1885, un projet plus ambitieux, subordonnant les législations cantonales à l'approbation du Conseil fédéral et prescrivant un ensemble de mesures tant sur la procédure électorale que sur l'octroi des droits politiques, s'enlise définitivement dans les débats parlementaires. Ce long processus donne lieu à plusieurs rapports du Conseil fédéral publiés dans la Feuille fédérale (1877, IV, pp. 411 sq ; 1878, II, p. 1041 sq. ; 1879, II, pp. 865 sq. ; 1881, I, pp. 336 sq. ; 1882 , III, pp. 1 sq. ; 1883 , IV, pp. 182 sq. ; 1885, IV, pp. 201 sq. ; 1886, I, pp. 129 sq.), rapports qui offrent un excellent aperçu des différents conflits suscités par des questions comme la durée et le lieu du scrutin, l'admission des bulletins imprimés, la tenue des registres électoraux et la détermination du domicile de l'électeur. 


\section{O S S I E R}

Histoire politique, histoire du politique II

Bernard Voutat

La codification du vote en Suisse (I848-I9I8)

fédéralisme

et construction du citoyen

30. Bulletin de l'Assemblée constituante du canton de Vaud, $B A C, 1861$, II, p. 273.

31. Procès verbal de la Commission du Conseil des États chargée d'examiner le projet de loi sur les élections et votations fédérales ; Feuille fédérale, 1885, IV, p. 209.

32. Rapport du Président de la Commission du Conseil des États chargée d'examiner la question de l'entrée en matière sur le projet de loi concernant les élections et votations fédérales ; Feuille fédérale, 1886, I, p. 131.

33. Sur la question de l'interprétation des deux votes de 1875 et 1877 , cf. Message du Conseil fédéral du 2 juin 1882 concernant le projet de loi sur les droits politiques des citoyens suisses, Feuille Fédérale, 1882, III, pp. 2 sq. toriales» ${ }^{30}$. A l'inverse, Altweg, député radical au Conseil des États et farouche partisan de l'unification législative, déclare à propos de l'élaboration d'une loi sur les élections et votations fédérales, «qu'il ne peut prêter les mains à aucun compromis sur le terrain du fédéralisme, [car] un tel procédé constituerait un affaiblissement du sentiment national suisse ${ }^{31}$. Les citations confirmant cette opposition fondamentale pourraient être multipliées, tant elles fourmillent durant toute la période considérée, et pas seulement à propos de la question des droits politiques. De fait, le désir constant des députés radicaux de parvenir à une unification législative au plan national, pour garantir «la régularité des scrutins» et "l'égalité entre les citoyens suisses» se heurte au constat non moins récurrent «des habitudes invétérées (...) prouvant que les représentants de nos différentes contrées sont attachés avec une grande ténacité à leurs vues respectives, si divergentes l'une de l'autre ${ }^{32}$. Les compromis passés dans les deux projets de loi de 1875 et 1877 ont, aux yeux du Conseil fédéral, conduit à leur échec, ceux-ci étant à la fois combattus par les conservateurs fédéralistes, défavorables à une libéralisation de la clause du domicile en faveur des confédérés, et par les radicaux, partisans quant à eux d'une rupture avec les entraves traditionnelles que la législation de plusieurs cantons apporte au droit de vote $^{33}$. Et en effet, l'ultime tentative d'unification, qui se déroule sur près de dix ans, entre 1877 et 1886, aboutit, en fin de compte, à ce que les Chambres fédérales n'entrent pas en matière sur le projet de loi du Conseil fédéral. Altweg, rapporteur de la commission du Conseil des États conclut du reste de façon assez désabusée :

«La conciliation des vues contradictoires paraissant impossible, on en vint forcément à se départir de la voie indiquée par le Conseil fédéral [i.e. celle de l'unification]. Renonçant à combiner, par des sacrifices mutuels, les vues diamétralement opposées, on s'en tira par des concessions faites au cantonalisme. De cette tendance naquit un texte qui ne fut pas même du goût de son propre auteur. Tout en déterminant en principe le droit de vote des faillis et des assistés, la loi ajoutait que tous les cantons n'étaient pas tenus d'appliquer le principe ainsi posé. Et de même, tout en assignant un mode uniforme aux élections et votations fédérales, la loi n'avait garde de déclarer que les cantons n'étaient pas tenus de s'y confor- 
mer. Enfin, n'osant déclarer le vote ni facultatif ni obligatoire, la loi laissait aussi sur ce point le champ libre à la souveraineté cantonale» ${ }^{34}$.

Ces échecs successifs relèguent au second plan l'élaboration d'une législation nationale sur les droits politiques. Le débat se déplace peu à peu sur la protection des minorités à travers l'introduction du système proportionnel dans la loi fédérale de 1919, sans que soient touchées toutes les autres dimensions de la procédure électorale et du droit de vote ${ }^{35}$. Certes, à cette époque, les pratiques au sein des différents cantons se sont partiellement égalisées en ce qui concerne les scrutins fédéraux, en raison de la jurisprudence de l'Assemblée fédérale ${ }^{36}$, mais surtout d'une nationalisation, certes limitée, de la vie politique, consécutive à la création, à la fin du XIX ${ }^{\mathrm{e}}$ siècle, des partis politiques au plan fédéral ${ }^{37}$.

La question de la centralisation au niveau fédéral des normes juridiques sur le vote relève sans conteste de la construction conflictuelle du national en regard des transformations socio-démographiques qui caractérisent l'ensemble de la période. Il s'agit en effet de répondre au problème de l'intégration dans le cadre national d'une population toujours plus nombreuse qui se détache des liens sociaux traditionnels.

A titre indicatif, on peut remarquer que la population en Suisse passe de 2,4 millions en 1850 à 3,8 millions en 1910. Alors qu'au milieu du siècle $42 \%$ des Suisses vivent dans des agglomérations de moins de mille habitants. $52 \%$ dans des bourgs de taille moyenne et seulement $6 \%$ dans des villes de plus de 10000 habitants, soixante ans plus tard, ces trois catégories représentent alors respectivement $23 \%, 51 \%$ et $26 \%$. La population des huit plus grandes villes a quintuplé dans ce laps de temps. Trois d'entre elles (Zürich, Genève et Bâle) atteignent une taille respectable (soit 200000 pour la première et 130000 pour les deux autres). Au début du $\mathrm{XX}^{\circ}$ siècle se marque ainsi une distinction très nette entre une Suisse industrialisée en pleine transformation et une Suisse rurale plutôt statique. Évidement, ce processus s'accompagne d'un brassage considérable de la population. La part de celle-ci qui réside dans sa commune, voire son canton d'origine diminue constamment $^{38}$. Entre 1870 et 1880 , soit dans la période où la codification du droit de vote est traitée de façon quasi permanente en Suisse et dans les cantons, le nombre des citoyens habitant leur propre canton ne s'est accru que de 32456 (soit de 1,46\%), tandis que celui des personnes résidant dans un autre canton, les confédérés. a augmenté, lui, de 84250 (soit de $22.5 \%$ ). Durant cette période. la proportion des «bourgeois» (c'est-à-dire des habitants originaires de la collectivité en question), des citoyens vivant dans
34. Feuille fédérale, 1886, I. p. 131.

35. Cf. le Message du Conseil fédéral du 26 novembre 1918 à l'Assemblée fédérale concernant les élections au Conseil national d après lc principe de la proportionnalité. Fenille Fédérale. 1918. V. pp. 119 sq.

36. Un député conservateur du Conseil des États. Théodore Wirz. loue cette approche "pragmatique» en indiquant ses résultats positifs concernant la conservation. l'uniformité et le comptage des bulletins. le nomadisme électoral et la clause du domicile, la proximité du lieu de vote. l'admission du bulletin imprimé. etc. Féuille fédérale, 1886. I, p. 141.

37. S'agissant des scrutins relevant des prérogatives cantonales, il convient de relativiser cette égalisation, ne seraitce qu'en raison du maintien dans quelques cantons de la Landsgemeinde où, par définition. le vote s'effectue à main levée.

38. Roland Ruffieux, «La Suisse des radicaux", in Nouvelle histoire de la Suisse et des Suisses, Lausanne. Payot, 1986 (deuxième éd.), pp. 668 sq. 


\section{O S S I E R}

\author{
Histoire politique, \\ histoire du politique II \\ Bernard Voutat \\ La codification du vote en Suisse \\ (1848-1918) \\ fédéralisme \\ et construction du citoyen
}

une autre commune de leur canton, des confédérés et des étrangers passe de, respectivement, $54 \%, 29 \%, 11 \%$ et $6 \%$ à $49 \%$, $30 \%, 14 \%$ et $7 \%{ }^{39}$. Encore que ces chiffres masquent des différences considérables selon les lieux, au point que très tôt déjà, dans certaines communes industrialisées, les «bourgeois» sont largement minoritaires, alors qu'il leur incombe de gérer des biens communaux souvent très importants. Citant un rapport du Bureau fédéral de la statistique, le Conseil fédéral s'inquiète de ce que «les ressortissants d'autres cantons, dont le nombre a doublé depuis trente ans, ne jouissent que de droits restreints ; les ressortissants d'autres communes du canton eux-mêmes ne sont pas partout sur un pied d'égalité avec ceux de la commune en ce qui concerne l'administration publique ; or, les bourgeois ne forment plus même la majorité de la population ; enfin, ces diverses catégories d'habitants du même pays sont encore régies par des législations civiles différentes». Aussi, le Conseil fédéral conclut-il que «dans l'intérêt de la consolidation de nos institutions», il s'agit d'étendre le droit de vote, «d'éveiller l'intérêt politique partout et de faire de tous les habitants des citoyens actifs (...). Il n'y a aucun danger dans la participation des grandes masses aux affaires publiques (...), mais il y en a un à les exclure ${ }^{40}$. L'égalité des citoyens du point de vue des droits politiques s'avère ici conforme au projet national du Parti radical, mais aussi à des intérêts électoraux évidents, dans la mesure où la population flottante, compte tenu de ses caractéristiques sociales, constitue un électorat hostile aux formations conservatrices dont elle s'est géographiquement éloignée. C'est dans ce cadre qu'il faut donc replacer les débats sur la clause de domicile liée à la capacité électorale, y compris dans leurs dimensions les plus techniques où interviennent des distinctions juridiques subtiles entre séjour, établissement et domicile proprement dit, ou encore dans toute une série d'entraves vis-à-vis des confédérés, à qui certaines législations cantonales demandent de fournir des preuves (souvent impossibles à se procurer) de leur qualité de citoyen de leur canton et de leur commune d'origine.

Cependant, plus fondamentalement, les transformations que nous avons rapidement évoquées ci-dessus s'inscrivent dans un long processus de dissolution des liens communautaires traditionnels, qui affecte directement les conditions mêmes de la compétition électorale. C'est dire qu'il serait tout à fait réducteur de s'en tenir à l'enjeu - le respect de la structure fédéraliste de la Suisse versus la nécessité d'une centralisation au niveau national - tel qu'il est explicité par les adversaires ou les partisans de l'uniformisation de la législation en matière de droits politiques. Comme le relèvent François Masnata et Claire Rubattel, «parler du fédéralisme, c'est parler simultanément du discours tenu sur le fédéralisme, de la pratique du discours et de sa signification pour le système politique» ${ }^{41}$. De fait, la généralisation progressive du droit de suffrage, par delà les divergences d'interprétation dont ce dernier est l'objet 
de la part des libéraux, des radicaux et des conservateurs, contraint ceux-ci à s'assurer le contrôle d'une pratique susceptible d'asseoir leur position de pouvoir dans les cantons où ils sont hégémoniques, ou à entrer dans une compétition électorale dont ils espèrent sortir vainqueurs. En ce sens, ce sont moins les conceptions politiques qui s'affrontent à propos du fédéralisme, des particularismes locaux, de la démocratie et de l'État suisse que les usages différenciés du vote par ces entrepreneurs politiques - qui permettent de rendre compte des modes spécifiques par lesquels se constituent les régimes politiques cantonaux et les différents codes électoraux qui y sont en vigueur. C'est pourquoi la lutte à propos des droits politiques, dont l'enjeu est la définition même du citoyen, se produit en partie sur le terrain du fédéralisme. En somme, la non-nationalisation du vote en Suisse (sur le plan juridique et pratique) est pour une part liée à un héritage historique où les diversités cantonales constituent un fait matériel indiscutable, mais pour une autre part, sans doute capitale, à une lutte (dont les formes et l'intensité varient d'un endroit à l'autre) sur la maîtrise du processus électoral. L'opposition entre radicaux (partisans de la centralisation) et conservateurs (défenseurs des particularismes locaux) est en effet largement tributaire des ressources spécifiques que ces courants politiques ont mis en œuvre dans l'entreprise de captation des suffrages. Elle marque au fond une tension, dans la redéfinition du lien social qui s'opère à la faveur de la construction sociale du politique, entre «la territorialisation de l'expression électorale ${ }^{42}$ et l'individualisation de l'acte de vote.

\section{Figures du citoyen et vérité des urnes}

Lorsque Antoine-Élisée Cherbuliez ${ }^{43}$ publie son livre sur la démocratie, il comprend bien que, s'agissant des formes de gouvernement, la Suisse est «dans un état de transition, où l'effet des institutions antérieures se manifeste encore et neutralise celui des institutions actuelles». Fin observateur des réalités du pays, il entreprend une analyse comparée de la démocratie "pure», telle qu'elle se pratique au sein des Landsgemeinde, et de la démocratie représentative, qui gagne du terrain dans plusieurs cantons suisses. De la première, il retient surtout les «vertus» et «qualités» du peuple réuni en assemblée. Il semble que ce soit, dit-il, «un être différent,
42. Michel Offerlé, «Le vote comme évidence et comme énigme». Genèses, mai 1993, n² 12, p. 151.

43. Les citations qui suivent sont tirées de Antoine-Elisée Cherbuliez. De la démocratie en Suisse, Genève, 1843, vol. 2, pp. 131 sq. Né en 1797 , mort en 1869, Antoine-Elisée Cherbuliez, publiciste, juge, docteur en droit et avocat. enseigne l'économie politique, la statistique et le droit public à l'Académie de Genève, puis à l'École polytechnique de Zürich. Il est en outre membre correspondant de l'Académie des sciences morales et politiques de Paris. Élu au Conseil représentatif du canton de Genève en 1831 , à l'Assemblée constituante et au Grand conseil en 1842, il quitte toutes ses fonctions à Genève en 1846. au moment de la révolution radicale, en raison de ses orientations politiques fortement conservatrices. Cf. Marcel Godet, Henri Türler et Victor Attinger (sous la dir. de), Dictionnaire historique et biographique de la Suisse, Neuchâtel. Attinger, 1921-1934, vol. 1, p. 500. 


\section{O S S I E R}

\author{
Histoire politique, \\ histoire du politique II \\ Bernard Voutat \\ La codification du vote en Suisse \\ (1848-1918) \\ fédéralisme \\ et construction du citoyen
}

44. Cette argumentation générale est tirée d'un ensemble de travaux. dont nous nous inspirons largement ici. Outre ceux déjà cités, mentionnons notamment Michel Offerlé, Un homme, une voix? Histoire du suffrage universel. Paris, Gallimard, 1993 ; Les partis politiques, Paris, PUF, 1987 ; "Le nombre de voix". Actes de la recherche en sciences sociales, mars $1988, n^{\circ} 71 / 72$, pp. 5-21 :

«Éclats de voix. L'élection comme objet de science politique».

Regards sociologiques, no 7. 1994. pp. 63-74 : Alain Garrigou, «Le secret de l'isoloir". Actes de la recherche en sciences sociales, mars 1988, n'71/72. pp. 22-45 : Yves Déloye et Olivier Ihl. «Des voix pas comme les autres. Votes blancs ct votes nuls aux élections législatives de 1881". Revue française de science politique, vol. 41, $\mathrm{n}^{\circ} 2$. avril 1991, pp. 141-170; Daniel Gaxie. Explication du vote, Paris, Presses de la Fondation nationale des sciences politiques, 1985. doué de plus d'intelligence et de discernement, surtout plus impressionnable que ses parties intégrantes». Bien entendu, ajoute-t-il, les démocraties «pures» ont un gouvernement qui doit rendre compte de ses actes devant le peuple, mais «ce qui rend cette garantie inoffensive, c'est précisément la forme sous laquelle le peuple est appelé à exprimer sa volonté». Et il ajoute : «La masse [assemblée] devient plus malléable, plus susceptible d'entraînements, plus accessible à toutes les influences extéricures (...). De là cet ascendant que les êtres prééminents par leur caractère, par leurs talents, par leur savoir, par leur position sociale, par l'autorité dont la loi les a revêtus obtiennent facilement sur une telle assemblée ; ascendant qui leur permet de la diriger, de la dominer, de lui faire partager leurs sentiments, de lui faire adopter leurs vues. Si les hommes du gouvernement de la Landsgemeinde ne peuvent rien sans le peuple et contre le peuple, ils peuvent l'amener à vouloir ce qu'ils veulent». Qui plus est, ce gouvernement n'a pas à craindre des assemblées extralégales, «car là où le peuple s'assemble constitutionnellement, là il ne songe point à s'assembler inconstitutionnellement». Par contraste, «le peuple a des qualités qui se perdent lorsqu'on le divise (...) en petites fractions», comme c'est le cas dans les démocraties représentatives, où, le plus souvent, «c'est isolément et sous le sceau du secret que chaque citoyen exerce son droit de suffrage. La volonté nationale n'y est que la somme des décisions de plusieurs assemblées locales, ou mêmes de votes individuels, c'està-dire de volontés partielles qui ont été déterminées par des intérêts locaux et par tous les motifs séducteurs auquel chaque citoyen est accessible». En définitive, «le peuple de la démocratie pure est un être moral, complet, unique, ayant sa vie tout à fait à lui, distincte de celle des individus». En revanche, «le peuple de la démocratie représentative n'est qu'une quantité abstraite, sans existence collective, un chiffre dont les unités sentent et agissent individuellement, comme si aucun lien ne les unissait entre elles, (...). Un monceau de sable, voilà ce qu'est devenu le peuple».

Ces remarques sur les pratiques «démocratiques» indiquent d'emblée l'une des dimensions sous-jacentes à la codification de l'acte de vote comme sanction d'une opposition entre des entrepreneurs politiques pourvus d'aptitudes inégalement réparties ${ }^{44}$ au travail de sollicitation des suffrages. 
On a déjà indiqué le contrôle notabiliaire qui pèse sur le processus électoral, mais il convient ici de préciser que celui-ci revêt des formes très diversifiées sur le territoire helvétique. Une multiplicité de facteurs interviennent à cet égard : intensité des mobilisations électorales liée à celle de la concurrence dans les scrutins ; configurations spécifiques des rapports de forces politiques au plan cantonal ; nature des transactions et accords électoraux effectués entre candidats; structures sociales (rurales versus urbaines) dans lesquelles se déroulent les campagnes électorales ; étendue des arrondissements électoraux. Dans les faits, nous retrouvons ici une dynamique que Max Weber a déjà fort bien identifiée, en énonçant que «l'administration des notables n'existe vraiment qu'aussi longtemps que des partis ne s'érigent pas en structures permanentes, ne se combattent pas et ne cherchent pas à s'approprier les fonctions. Dans le cas contraire, le chef du parti qui combat et triomphe (...) devient avec sa direction administrative un instrument de domination, malgré le maintien de toutes les formes de l'administration antérieure. C'est là une forme assez répandue de la destruction des «anciens rapports» ${ }^{45}$. De 1848 à 1917, Erich Gruner recense 1324 scrutins, dont 1088 au premier tour. La participation électorale, très variable selon les lieux et les époques, n'a pas partout la même signification. Tantôt elle est liée à l'autorité de notabilités locales pouvant s'appuyer sur des liens communautaires forts, parfois reliés par l'Église catholique. Tantôt elle est tributaire de mobilisations, dont l'ampleur dépend fortement de l'intensité de la lutte dans les scrutins, faible dans un tiers des cas, moyenne dans l'autre tiers et forte dans le dernier tiers. S'agissant des incitations à voter, Gruner montre également que la constitution des entreprises politiques suit des rythmes différenciés. Au niveau national, elle se produit vers la fin du XIX siècle (Parti socialiste en 1888 ; Parti radical en 1894 ; Parti conservateur catholique en 1894, puis 1912), mais reste limitée. Les étiquettes nationales recouvrent en effet des réalités souvent hétérogènes, qui renvoient à des situations locales distinctes.

Dans les cantons conservateurs de la Suisse centrale, qui forment souvent des arrondissements à un seul siège, l'appropriation des suffrages suit une logique qui relève de la «ratification de l'autorité légitime»selon l'expression de Siegfried que Cherbuliez n'aurait certainement pas reniée. Dans les cantons où l'antagonisme entre radicaux et libéraux et/ou conservateurs est précoce (Vaud, Genève, Zürich, Neuchâtel, Bâle, Berne notamment) se constituent des comités électoraux plus ou moins permanents, sous la forme d'abord des Associations patriotiques créées par les radicaux. Dans certains cantons marqués par des luttes électorales de très forte intensité se mettent en place des organisations secrètes, centralisées et hiérarchisées, encadrant l'électorat d'une main de fer, comme à Fribourg où les conservateurs parviennent à contrôler la quasi totalité des électeurs ${ }^{46}$.
45. Max Weber, Économie et société, op. cit., Vol. 1, p. 380.

46. Sur ce canton, voir la remarquable ćtude, inspirée des travaux de Maurice Agulhon, de Pierre-Philippe Bugnard, Le machiavélisme de village : la Gruyère face à la République chrétienne de Fribourg (1881-1913), Lausanne, Le front littéraire, 1983, pp. $88 \mathrm{sq}$. Dans cette étude, l'auteur mentionne l'existence d'une organisation se proclamant secrète et permanente, parallèle au parti conservateur officiel, chargée de dresser une liste de tous les électeurs du district de la Gruyère, et disposant pour ce faire d'hommes de confiance, appelés aussi «agents secrets». 


\section{O S S I E R}

Histoire politique, histoire du politique II

Bernard Voutat

La codification du vote en Suisse (I848-I9I8)

fédéralisme

et construction du citoyen

47. $35 \%$ concernent des falsifications des résultats, $26 \%$ des «influences frauduleuses sur la formation de l'opinion», $21 \%$ des irrégularités dans le déroulement du processus électoral et $18 \%$ des manipulations du registre électoral. Erich Gruner, Die Wahlen in den Schweizerischen Nationalrat (1848-1919), vol. 1, op. cit., p. 1031.

48. Message du Conseil fédéral du 24 juin 1872 touchant un projet de loi sur les élections et les votations fédérales, Feuille Fédérale, I, 1872, p. 825 .
La diversité des conditions de la compétition électorale se remarque également dans la géographie des fraudes. Dans certains cantons à fort antagonisme électoral, celles-ci sont assez fréquentes (Berne, Fribourg, Argovie, Genève). Elles constituent parfois la règle, comme au Tessin où, dans un contexte de luttes intenses entre conservateurs et radicaux caractérisées par des pratiques frauduleuses d'envergure (manipulation de registres électoraux, brigues et achats de voix, «nomadisme» électoral, irrégularités nombreuses dans le comptage des suffrages, violations répétées du secret du vote), les élections sont régulièrement contestées, et ceci jusque dans les premières années du $\mathrm{xx}^{\mathrm{e}}$ siècle. Ailleurs, une «morale électorale»s'est imposée plus précocement, comme dans les cantons marqués par les radicaux (Vaud, Neuchâtel, Zürich, Bâle-ville, St-Gall, notamment), où les contestations des scrutins se font extrêmement rares dès le milieu du XIX ${ }^{e}$ siècle. Certes, les distinctions entre irrégularité, fraude et travail toléré de propagande ne sont pas toujours faciles à établir. Sur l'ensemble de la période et pour les 1324 scrutins considérés, Gruner dénombre toutefois 170 recours et constate une nette diminution des pratiques irrégulières avec le temps ${ }^{47}$.

Sur le plan de la codification juridique, la question des fraudes est cependant évoquée de façon récurrente (notamment à travers les cas où elle est particulièrement prégnante) et l'on voit se dessiner progressivement une représentation du vote comme étant un droit du citoyen que ce dernier doit pouvoir exercer individuellement à l'abri des pressions de toutes sortes. La garantie du secret du vote constitue donc, dans cette optique, l'élément central des dispositions réglementant le déroulement proprement dit du scrutin. Introduit explicitement dans la loi fédérale de 1872, ce principe se heurte alors à des pratiques distinctes, on l'a vu, dans les différent cantons, notamment dans ceux où l'élection se déroule à main levée au sein des Landsgemeinde. Or, la reconnaissance par le Conseil fédéral du vote secret comme «disposition essentielle de la procédure électorale ${ }^{48}$ ne fait en somme que sanctionner un rapport de forces acquis sur le plan cantonal et dans les chambres fédérales où les radicaux dominent nettement : «Dans la plupart des cantons, qui représentent au moins $80 \%$ de la population suisse, le vote secret a été substitué à l'ancien vote à main levée pour toutes les votations et élections canto- 
nales, et personne ne songe à en revenir à l'ancien système. On admet généralement que l'indépendance de l'électeur ne peut être garantie que par le vote au bulletin, et que le citoyen dépendant par son état de fortune et sa position sociale doit réclamer ce mode de votation si, du moins dans l'exercice de ses droits politiques, il veut être l'égal de celui d'après lequel il est obligé de se diriger dans sa vie ordinaire. En effet, avec le vote à main levée, c'est en réalité le maître qui commande à ceux qu'il emploie, ou bien on voit se substituer aux influences matérielles des influences morales qui ne sont pas moins funestes à l'indépendance de l'individu ${ }^{49}$.

Le principe du vote secret constitue alors l'une des principales dimensions intervenant à l'occasion des nombreux débats sur l'élaboration d'une éventuelle législation fédérale sur les droits politiques, qui se substituerait à celles en vigueur dans les différents cantons. Ainsi, en 1875, l'Assemblée fédérale adopte le postulat suivant : «Le Conseil fédéral est invité à examiner si, en vue d'assurer la sincérité du vote et de fournir un moyen de contrôle efficace, il n'y a pas lieu de compléter la législation fédérale, en introduisant un mode de procéder uniforme dans tous les cantons et à présenter, cas échéant, un projet de loi dans ce sens» ${ }^{50}$. Dans son message, le Conseil fédéral, après un examen des lois et pratiques cantonales réglementant la procédure électorale, prend acte de la très grande diversité des moyens mis en œuvre dans les cantons pour «assurer l'indépendance de l'électeur» et «empêcher les fraudes électorales». Il juge alors que la procédure électorale «devrait être entourée des formes qui donnent le plus de sécurité (...), dans la mesure où le peuple suisse a droit à ce que l'expression de sa volonté soit pure et vraie et que des règles sévères et une surveillance stricte excluent, dans la mesure du possible, les abus, les falsifications et autres inconvénients» ${ }^{51}$.

Qu'en est-il de la pratique ? Selon la plupart des gouvernements cantonaux, le secret du vote est respecté dans les faits. Les procédures électorales sont cependant très variables. Cette diversité touche notamment la composition du bureau et la nomination des membres, ainsi que la vérification du droit de vote, qui s'opère tantôt par appel nominal, tantôt au moyen d'une carte de légitimation. Les bulletins de vote sont distribués soit au domicile, soit dans l'assemblée électorale même. Leur confection s'accomplit parfois dans la salle de vote, mais dans un lieu

\section{Ibid.}

50. Message du Conseil fédéral du 27 novembre 1877 à la haute Assemblée fédérale concernant des dispositions complétant les lois fédérales du 19 juillet 1872 sur les élections et votations fédérales et du 17 juin 1874 concernant les votations populaires sur les lois et arrêtés fédéraux, Feuille Fédérale, 1877, IV, p. 411.

51. Ibid., p. 430. 


\section{O S S I E R}

Histoire politique. histoire du politique II

Bernard Voutat

La codification du vote en Suisse (1848-1918) fédéralisme

et construction du citoyen réservé à cet effet, ou dans un «compartiment» séparé de celle-ci, soit un isoloir. Les bulletins sont tantôt imprimés, tantôt remplis par l'électeur lui-même ou par une tierce personne de son choix. Leur dépôt s'effectue entre les mains du scrutateur ou dans une urne placée sous les yeux de tous les électeurs. L'électeur, le plus souvent, a l'obligation de déposer son bulletin personnellement dans l'urne, mais il a parfois la faculté de le remettre à un tiers. Enfin, le lieu du scrutin varie considérablement et se situe soit à la commune, voire dans une section de commune, soit alors au chef lieu du cercle électoral. Certains cantons, enfin, rendent le vote obligatoire, d'autres facultatif.

Le gouvernement lucernois précise que, compte tenu de la procédure en vigueur dans le canton, il ne dépend que de l'électeur de maintenir son vote secret, même s'il a loisir d'en rendre le contenu public. Cette garantie est illustrée à ses yeux par une affaire de pression sanctionnée par une poursuite pénale, où un employeur avait contraint des ouvriers à présenter ouverts leurs bulletins avant que ceux-ci ne soient déposés. D'autre part, le canton de Schwytz déclare avoir renoncé à numéroter les bulletins de façon à ce que le fonctionnaire chargé de leur distribution ne puisse identifier les votes des électeurs. Le gouvernement fribourgeois précise en outre que l'indépendance de l'électeur est assurée du fait qu'il n'a pas l'obligation d'inscrire son vote sur les bulletins officiels, mais qu'il peut utiliser le bulletin de son choix pourvu que celui-ci ait le même format et le même papier. A Soleure, il n'existe pas de bulletin officiel. Chaque électeur peut faire usage d'un bulletin de son choix, à condition que le papier soit blanc et qu'il ne porte pas de numéro, afin que les partis ne puissent pas exercer un contrôle quelconque. Par ailleurs, les bulletins imprimés sont admis, mais il est interdit de les distribuer aux électeurs à l'intérieur du local de vote. A Neuchâtel, la procédure prévoit la distribution d'un bulletin officiel (vierge) lorsque le citoyen pénètre dans la salle de vote. Celle-ci est disposée de façon à ce qu'il puisse écrire son vote en toute liberté et secrètement, derrière un paravent ou dans un compartiment séparé. A Genève, c'est le système de l'«estampille» qui est en vigueur. Ainsi, l'électeur a tout loisir de marquer le bulletin de son choix, le secret étant garanti par le fait que chaque bulletin, une fois plié, est de couleur et d'apparence uniforme. Quant au bulletin imprimé (en usage dans quelques cantons seulement), son admission reste controversée. Bien que la loi fédérale prescrive que le vote doit être «écrit», certains parlementaires estiment qu'il devrait l'être personnellement. Se situant explicitement sur le terrain de l'«indépendance de l'électeur», le Conseil fédéral juge alors que celle-ci n'est pas mise en danger dès lors que chaque citoyen a la possibilité soit d'écrire lui-même son bulletin à son gré, soit de faire usage d'un bulletin imprimé, qu'il peut par ailleurs modifier. 
Bref, après examen des législations et des technologies entourant le secret et l'authenticité du vote, le Conseil fédéral estime que des garanties sérieuses existent en la matière, qui ne rendent pas nécessaire l'élaboration de dispositions fédérales, par ailleurs difficilement conciliables avec des habitudes cantonales fort diversifiées. A ses yeux, les procès-verbaux des élections et des votations fédérales dressent «un tableau fidèle» $^{52}$ de la réalité. Sans doute cet accord entre les dispositions juridiques et la réalité n'est pas aussi parfait que ne le laisse entendre le Conseil fédéral à l'occasion de ce débat parlementaire, tant il est vrai que le droit est ici en retard sur des pratiques hétérogènes qu'il ne sanctionne qu'en partie. Du reste, le problème resurgit par la suite, notamment lorsque sont traitées de graves affaires de fraudes ${ }^{53}$. Mais l'essentiel à nos yeux relève plutôt de la représentation du citoyen et de la démocratie qui s'institutionnalise dans le droit et se solidifie dans le dispositif matériel par lequel est aménagé le vote (bulletin, enveloppe, isoloir, urne, etc.). Selon le Conseil fédéral, «la question de forme est ici d'une importance essentielle. Le droit de vote resterait lettre morte sans le secours de dispositions assurant son exercice (...) et c'est sur le terrain de l'accord parfait du droit de vote avec les conditions et les formes de sa manifestation que nous cherchons à consacrer l'uniformité dans la procédure électorale (...), sans laquelle une opération électorale ne saurait prétendre être l'expression de la vérité» ${ }^{54}$. Cette vérité, désormais, se mesure à la «liberté d'action»du citoyen qui, «sans être obsédé par les influences étrangères», est à même de «déposer dans l'urne le suffrage qui correspond le mieux à sa volonté personnelle»s5. Dans cette optique, «le moment le plus important (...) est celui où l'électeur a reçu son enveloppe officielle dans le local électoral et se dispose à donner son suffrage» ${ }^{56}$.

Il reste que si le secret du vote est progressivement assuré dans les faits, le citoyen doit encore se déplacer aux urnes. A mesure en effet que l'individualisation de l'acte de vote s'accomplit, les modalités communautaires qui président au déroulement de l'opération électorale constituent un obstacle à une participation politique élargie. C'est dans ce contexte qu'interviennent les mesures destinées à réduire le temps nécessaire à l'accomplissement du vote et à rapprocher l'urne de
52. Ibid., p. 435.

53. Cf. à ce sujet, notamment le Rapport du Conseil d'État du canton du Tessin au sujet des élections au Conscil national dans les $40^{\circ}$ et $41^{\mathrm{C}}$ arrondissements électoraux fédéraux du 22 novembre 1881 , Feuille fédérale, IV, 1881, pp. $678 \mathrm{sq}$. ; le Rapport de la commission spéciale au sujet des élections au Conseil national dans le $40^{\mathrm{e}}$ arrondissement électoral fédéral (Tessin) du 2 juin 1882 , Feuille fédérale, III, 1882, pp. 137 sq.

54. Message du Conseil fédéral à l'Assemblée fédérale du 30 octobre 1883 concernant le projet d'une loi fédérale sur les élections et votations fédérales, Feuille fédérale, 1883, IV, pp. 192-193.

55. Ibid., p. 199.

56. Message du Conseil fédéral à l'Assemblée fédérale du 24 février 1885 concernant l'insertion de dispositions pénales dans le projet de loi sur les élections et votations fédérales, Feuille fédérale, 1885, I, p. 401. 


\section{O S S I R}

Histoire politique, histoire du politique II

Bernard Voutat La codification du vote en Suisse (I848-1918) fédéralisme et construction du citoyen

57. Message du Conseil fédéral du 27 novembre 1877, Feuille Fédérale, 1877, IV, p. 438. l'électeur. Alors qu'au milieu du XIX ${ }^{\mathrm{e}}$ siècle, il n'est pas rare qu'une élection prenne un temps considérable - qui peut aller jusqu'à plusieurs semaines s'agissant, par exemple, de la nomination au sein d'une assemblée des cent représentants au législatif communal de Lausanne (élus au scrutin uninominal à quatre tours !) - ce temps se réduit à quelques minutes à la fin du siècle, là où le citoyen a la possibilité de se rendre individuellement au moment de son choix dans un local de vote. De même, le lieu du scrutin est-il associé à ce processus d'individualisation du vote. Le vote à la commune, voire dans une section de commune, s'il est destiné à diminuer le trajet entre l'urne et le domicile, limite du même coup l'influence qu'exercent les notables au sein de larges assemblées. Encore que l'argument inverse ait pu être invoqué dans le canton de Genève, qui ne connaissait en 1872 qu'un seul lieu de vote pour l'ensemble du canton, contraignant certains électeurs à parcourir près de vingt kilomètres. L'argumentation en faveur de ce système, qu'il convient de rapporter au fait que la campagne est tenue par les conservateurs, est d'ailleurs fort significatif de l'enjeu que constitue cet aspect du dispositif électoral, qui permet selon le Conseil d'État genevois «d'assurer la liberté des citoyens, en les éloignant des influences locales» ${ }^{57}$. Du reste, la multiplication, quelques années plus tard, des lieux de vote à Genève manifeste l'effectivité du processus d'individualisation du citoyen, qui, par delà la spécificité des situations locales, est progressivement consacrée par le droit.

Territorialisation du vote et individualisation du citoyen, telle paraît bien être la trame de la construction de l'espace politique en Suisse. La tension entre ces deux dimensions s'inscrit dans le processus de codification juridique de l'acte de vote où l'élaboration des règles de droit en la matière prolonge et sanctionne des rapports de forces politiques hétérogènes durant toute la période. C'est dire que le fédéralisme, comme du reste la «démocratie suisse», ne contiennent pas en euxmêmes leur propre définition, mais sont des institutions dont les propriétés ne peuvent être pleinement saisies qu'une fois rapportées à leur genèse. Or, la question des spécificités helvétiques pose d'emblée un problème à l'analyste. Comme le soulignent François Masnata et Claire Rubattel, «de nombreux politologues, après avoir affirmé au départ de leur recherche le «caractère parti- 
culier» de la Suisse, s'étonnent en conclusion que la Suisse a un caractère particulier» 58 . Pour éviter «le piège de l'exotisme», qui conduit à réduire les différences empiriques constatées à des différences de «nature», il faut en rendre compte «en analysant la spécificité d'une histoire collective» tout en la considérant comme «un cas particulier du possible (...), c'est-à-dire comme un cas de figure dans un univers fini de configurations possibles $(. .)^{59}$. C'est en fonction de ce point de vue que nous avons organisé notre raisonnement, et que nous nous sommes efforcé d'identifier les logiques sousjacentes à la codification juridique de l'acte de vote en Suisse durant la deuxième moitié du XIX ${ }^{\mathrm{e}}$ siècle. $\mathrm{Si}$, incontestablement, le fédéralisme, comme système de classement du monde social, et les rapports de forces politiques qui y sont associés, introduisent de la singularité dans ce processus de construction du citoyen suisse, notre enquête montre néanmoins que ce dernier n'est pas indépendant d'une logique invariante issue des usages du vote, de la pratique de conquête des suffrages et donc de l'émergence progressive d'un ordre politique désencastré du social, marquant, selon l'expression de Max Weber, «la destruction des anciens rapports».

$\mathrm{Si}$, d'un côté, «une institution est le résultat d'engagements et d'activités hétérogènes jamais complètement voulu par quiconque non plus que jamais contrôlé par personne ${ }^{60}$, de l'autre, elle ne peut être saisie en dehors du système de contraintes génériques par rapport auquel elle se constitue comme une «réponse» ou une «solution» provisoire, plus ou moins contingente. En ne tenant compte que des invariants, outre que l'on s'expose à toutes sortes d'anachronismes, voire à «naturaliser» l'ordre social, on s'interdit par ailleurs de comprendre la façon dont ils sont gérés dans un contexte historique particulier. En retour, se limiter aux représentations que les acteurs se font des institutions qu'ils construisent ne permet pas de saisir ce par rapport à quoi ces institutions prennent sens ${ }^{61}$. C'est bien pourquoi une socio-histoire du politique se doit de «saisir l'invariant dans la variante observée» ${ }^{62}$. C'est ainsi que le vote s'inscrit comme une modalité et une forme spécifiques de la «décision collective», dont les caractéristiques élémentaires, loin de se limiter à une dimension purement technique, ne se comprennent qu'une fois rapportées aux relations sociales dont elles procèdent ${ }^{63}$.
58. François Masnata et Claire Rubattel, Pouvoir suisse, op. cit., p. 13.

59. Pierre Bourdieu, Raisons pratiques, Paris, Seuil, 1994, p. 16.

60. Bernard Lacroix, Jacques Lagroye, Le Président de la République. Usages et genèse d'une institution, Paris, Presses de la Fondation nationale des Sciences Politiques, 1992, p. 9.61. Cf. François Masnata, Le politique et la liberté. Principes d'anthropologie politique, Paris, L'Harmattan, 1990, pp. $109 \mathrm{sq}$.

62. Pierre Bourdieu, Raisons pratiques, op. cit., p. 17.

63. Sur cette question, voir la contribution d'Alain Garrigou, «La construction sociale du vote. Fétichisme et raison instrumentale», Politix, n²2, deuxième trimestre 1993 , pp. 16 sq. 